\title{
Particulate steroids in axial spinal blockade and the increasing role of patient consent: Les grains de sable dans l'engrenage.
}

\begin{tabular}{|r|l|}
\hline Journal: & British Journal of Pain \\
\hline Manuscript ID & BJP-19-0099.R2 \\
\hline Keywords: & Back Pain, corticosteroids, epidural injection, Neck Pain, side effects \\
\hline Abstract: & $\begin{array}{l}\text { The complexity of modern medical practice is such that it is very unlikely } \\
\text { that on any single issue we can give a definitive answer in any } \\
\text { circumstance, and in our view the medical debate as to the use of } \\
\text { particulate corticosteroid medicine in axial spinal blockade is one such } \\
\text { argument. The medical discussion of the use of particulate } \\
\text { corticosteroids has to be set against the uncertain risk and benefits of } \\
\text { axial spinal procedures in which the drugs are utilised, and in which the } \\
\text { most likely catastrophic complication may occur with their use, and then, } \\
\text { as the law now demands, involve the patient in the relevant consenting } \\
\text { issues. }\end{array}$ \\
\hline
\end{tabular}

\section{SCHOLARONE Manuscripts}




\section{Introduction}

Medical treatments have never operated in the isolation of a simple doctor-patient relationship. Many other external factors need to be considered including relevant clinical evidence guidelines and medicolegal issues and specifically those of consent. The use of particulate steroids in axial spinal procedure is a case in point. While this treatment is commonly used, considerable doubts remain as to its effectiveness. Moreover the question arises as to whether the patient fully understand the risks and benefits of using the intended medicines and the procedure.

The use of particulate steroid in axial spinal blockade is a situation in which the specific particulate properties of the medicine may cause such catastrophic longterm neurological problems through damage to the spinal cord via ischaemic mechanisms. These catastrophic complications may follow even the most expertly performed procedure and frequently lead to a legal claim, based not on the standard of practice (which may have been immaculate as complications occur in even the most skilled hands) but instead on a failure to obtain adequate informed consent. In such situations patients or clients are often heard to say something like 'if I had known this might occur I would never have agreed to this treatment'. The ensuing investigation leaves a clinician involved in such a situation bewildered, and facing years of legal scrutiny and uncertainty.

This brief paper discusses the intersection of the major issues that have to be considered when prescribing particulate steroids in axial spinal procedures.

The first question is whether there are specific risks associated with particulate steroids for axial spinal procedures which can be fully mitigated by the use of alternative and equally efficacious medicines? The second question relates to the fundamental issue of whether such axial spinal procedures in general should be performed in the first place. Thirdly, the question is whether the patient fully understands the complexity of the previous two issues and can give meaningful consent for the procedure and the particulate medicine; in particular, whether the patient has really given consent for the intervention if they understand that the benefits are likely to be at best modest and transient with the use of particulate steroids, and accompanied by an admittedly small risk of major catastrophic complications.

We will not try to reproduce all the evidence which is available concerning the possible complications of particulate steroids in axial spinal blockade in the literature. This has been more than adequately covered, ${ }^{1}$ but we will focus instead on addressing the intersection of the three questions given above

The evidence base of particulate steroids causing catastrophic neurological damage in axial spinal procedures.

Scientific studies demonstrate that following arterial injection, particulate corticosteroid preparations may form aggregates which may act as emboli to block 
small terminal arterioles in the brain and spinal cord. Methylprednisolone has the largest particle size, triamcinolone is intermediate, and betamethasone has the smallest. Dexamethasone is a solution and does not form particles or aggregates. ${ }^{2}, 3$

Arterial injection of particulate methylprednisolone has been shown to cause severe neurological consequences such as unconsciousness and cerebral haemorrhage. ${ }^{4}, 5$ Particulate corticosteroid preparations have been associated, in published case reports, with neurological damage and vascular complications in humans. ${ }^{6}, 7,8$

As a result of this evidence clinicians instead have turned to using non-particulate (liquid) corticosteroids for axial spinal procedures in an attempt to reduce the chance of such complications but concerns were raised that water soluble corticosteroids were perhaps less clinically efficacious than particulate corticosteroid formulations. ${ }^{9}$ Overall the results following longer-term clinical outcomes after treatment comparing particulate and liquid corticosteroid preparations are equivocal. Park et al [2010] demonstrated that triamcinolone (a particulate preparation) reduced the visual analogue score (VAS) better than dexamethasone (a liquid). ${ }^{10}$ However, no difference was found between the groups using the McGill Pain Questionnaire or the Oswestry Disability Index. Kim \& Brown [2011] found dexamethasone phosphate solution comparable to particulate methylprednisolone acetate in the treatment of lumbar radiculopathy, although dexamethasone demonstrated a trend toward less pain relief and a shorter duration of clinical efficacy. ${ }^{11}$ Feeley et al (2017) concluded that particulate steroids are not demonstrably better in relieving pain compared to their non-particulate counterparts and, in view of the safety concerns of particulate steroids, suggested it may be prudent to switch to non-particulates, or at the very least the dangers and alternatives should be flagged with the patient group as part of a shared decision-making process (our italics). ${ }^{12}$

In a large retrospective observational study $(3,645$ lumbar transforaminal epidural steroid injections (TFESIs) performed on 2,634 subjects for radicular pain with or without radiculopathy) there was no evidence that dexamethasone was less effective in pain relief and functional improvement than particulate corticosteroids. ${ }^{13} \mathrm{~A}$ high quality trial on 78 consecutive subjects found that both triamcinolone and dexamethasone resulted in significant improvements in pain and function at 2 weeks, 3 months, and 6 months, but without clear differences between groups, although patients in the dexamethasone group required slightly more injections than the triamcinolone group in order to achieve the same outcomes. ${ }^{14}$

Using the American Society of Anaesthesiologists Closed Claims database, epidural corticosteroid injections accounted for $83 \%$ of injections and $40 \%(114 / 284)$ of all chronic pain management claims. ${ }^{15}$ Significant nerve injury (with seven claims of quadriplegia/paraplegia) was also observed in 28 epidural corticosteroid claims but it was not specified how many related to use of particulate corticosteroid. Claims related to procedures performed at the level of the cervical spine comprised $22 \%$ of all claims related to chronic pain treatment, which suggested a relative increased risk in 
this area of the spine. ${ }^{16}$ Of the 64 cases, there were nine $(14 \%)$ cervical procedures associated with spinal cord infarction or stroke after intra-arterial injection. In five of these cases, spinal cord infarction followed cervical transforaminal injection of particulate corticosteroid. In three other claims, cervical transforaminal injection of particulate corticosteroid resulted in stroke, presumably by injection into the vertebral artery.

Concerns have also been raised whether corticosteroid formulations used for epidural administration should contain a preservative or not, although no preparations currently in the UK have a marketing authorization for epidural administration, so use by this route is currently an 'off-label' indication. There are, however, no documented adverse events from the placement of preservative-containing corticosteroids in the epidural space (although neurological injuries have been documented when the preservatives have been injected inadvertently by the intrathecal route). There have also been multiple cases of fungal infections caused by epidural and paraspinal injection of a contaminated glucocorticoid product in the US. Clinicians who elect to use preservative-free corticosteroids must carefully weigh the risks and benefits and address sterility concerns. ${ }^{17}$

There also remains controversy as to any additional benefit from adding a corticosteroid to local anaesthetic for epidural administration. In a recent systematic review, high-quality evidence from multiple high-quality randomized controlled trials identified that the combination of local anaesthetic with corticosteroid was effective in managing chronic spinal pain. Local anaesthetic with corticosteroid and local anaesthetic alone were equally effective in a range of spinal conditions except in disc herniation, where the combination was superior. ${ }^{18}$

Based on the medical evidence the British Pain Society (BPS) and Faculty of Pain Medicine of the Royal College of Anaesthetists (FPMRCA) produced a Consensus Statement on the use of Corticosteroids for Neuraxial Procedures in the UK. They decided that due to the disproportionately large number of case reports of neurological complications related to the cervical region compared to the lumbar region, and also distinguishing between the interlaminar region compared to transforaminal injection, a case could be made for a clinician using different drugs in different regions. This position statement did, however, emphasise patient involvement in the decision-making progress. ${ }^{19}$

The conclusions of the BPS/FPM position statement are given below:

1. Particulate steroids must not be used for transforaminal cervical epidural injections on the basis of the risk of rare but catastrophic complications.

2. Whilst definitive recommendations cannot be given for the choice of soluble or particulate steroid for injections in interlaminar cervical epidurals, clinicians should be aware that serious neurological complications can still occur.

3. Whilst definitive recommendations cannot be given for the choice of soluble or particulate steroid for injections in epidurals undertaken in other areas of the 
spine (thoracic, lumbar and caudal), clinicians should be aware that serious neurological complications can occur with any route of administration, particularly if there is a history of previous spinal surgery.

4. Steroid preparations for epidural administration may carry a small risk of neurotoxicity with inadvertent intrathecal injection, due to the preservative preparation used. The clinician should carefully consider the formulation used.

5. The doctor must follow current GMC guidance on consent and record the discussion process. The discussion should ideally occur on an occasion prior to the procedure, as well as at the time of the procedure, to allow time for reflection.

6. The consent process should include discussion and documentation regarding indications, efficacy, safety and alternative treatments.

7. The use of corticosteroids in epidural injections is an indication that is outside the marketing authorisation (product licence). This information should also be incorporated into the consent process and documented in the medical records. The issue arises with the above position statement, of course, that if something is more likely to cause catastrophic neurological injury in the cervical region, why should particulate corticosteroids still be used at all in the lumbar region, even if the everpresent risk might be reduced in the lumbar region? It was accepted, however, that the risk of neurological injury was raised by the presence of altered anatomy (say in the case of prior spinal surgery).

What needs to be considered at this point in the discussion is point 6 in the FPM/BPS guidance, in order to focus on the role of the patient in the decision-making process. In particular, is the clinician entitled to make such a decision on behalf of the patient or should the patient have a greater role in the consenting process, in a scenario where there is likely a raised chance of a rare catastrophic neurological complication with particulate corticosteroid risk, compared to virtually no chance of such a complication with the use of non-particulate steroids?

\section{Principles of consent}

The GMC has provided a framework for consent which all medical practitioners are expected to be familiar with and to follow ('Consent: patients and doctors making decisions together' GMC 2008). ${ }^{20}$ These principles have been strengthened in the draft updating new guidelines which are out for consultation. ${ }^{21}$ Briefly, the principles include the following:
a. listen to patients and respect their views about their health
b. discuss with patients what their diagnosis, prognosis, treatment and care involve
c. share with patients the information they want or need in order to make decisions




\title{
d. maximise patients' opportunities, and their ability, to make decisions for themselves \\ e. respect patients' decisions.
}

There is recognition of the complexity and uncertainty in medical information and practice and the difficulty in applying appropriate information to a specific individual. In the past, the Bolam and Bolitho tests have provided the standard by which the Courts assess whether medical performance is or is not negligent. The Bolam test states that a doctor is not negligent and therefore has performed to an acceptable standard if he or she has acted in accordance with a practice accepted as proper by a responsible body of medical practitioners in that field of medicine. ${ }^{22}$ Bolitho extended that principle to allow the Courts to reject a practice supported as proper by medical opinion if the court considered that it did not withstand logical analysis. ${ }^{23}$ However, in relation to consenting a patient and warning of the risks of treatment, the Supreme Court ruling in Montgomery (2015) has retrospectively superseded the older tests by imposing on a doctor the duty: ${ }^{24}$

\author{
"to take reasonable care to ensure that a patient is aware of any \\ material risks involved in any recommended treatment, and of \\ any reasonable alternative or variant treatments."
}

For these purposes a risk is material if a reasonable person in the patient's position would be likely to attach significance to the risk, or if the doctor was, or should reasonably have been, aware that the particular patient would be likely to attach significance to it.

Therefore, doctors must now ask themselves three questions (Sokol 2015): ${ }^{25}$

1) Does this patient know about the material risks of the treatment I am proposing?

2) Does this patient know about reasonable alternatives to this treatment?

3) Have I taken reasonable care to ensure that this patient actually knows this?

Within the consenting process relating to the use of steroids in central neuraxial procedures the following practices need to be demonstrated to have been performed:

1) There needs to be a provision of information in a manner which will allow for understanding by this particular patient. The complexity of information and issues that need to be considered means that in practice the prior provision and consideration of written information (for example a patient information leaflet, or similar, and a copy of the clinic letter) followed by a subsequent discussion is most likely to achieve this. This consenting process will take time and will 
usually require more than one occasion and should not be rushed. The fact that it has taken place, its duration and the key points discussed must be documented.

2) There needs to be a demonstration that those particular factors that are likely to matter to this particular patient have been discussed. Risks or complications which may not concern another patient may be very important to this one.

3) There needs to be a demonstration that care was taken that this patient understands the implications of what is being done or suggested, what alternative or variant treatments exist, and the implications of not going ahead with the proposed treatment pathway.

It should also be noted that a patient who has been consented for treatment by Doctor A but on the day of the procedure is presented with Doctor B may have grounds for complaint should there be an adverse consequence to the procedure, if the choice of doctor was material to the giving of consent. Therefore, where there is such a change of personnel, it is important that the patient is again consented and the further consent properly documented prior to the procedure. ${ }^{26}$

Medical consenting issues related to the use of epidural corticosteroids injections for pain:

In guiding the development of a patient information leaflet and guiding the discussion and the consenting with the patient, in our view the following need to be discussed. (See also Appendix 1.)

- The use of corticosteroids in epidural injections is routine and probably does increase their efficacy compared to injections without corticosteroids.

- The use of particulate corticosteroids, particularly in the cervical region, is associated with more case reports of catastrophic complications. In contrast, the use of non-particulate corticosteroid preparations such as dexamethasone has been reported in world literature to be associated with less than a handful of such catastrophic complications.

- Experience of the use of non-particulate steroid preparations is much more limited than particulate, and there are some concerns about the possible reduced efficacy of non-particulate corticosteroid preparations compared to particulate preparations. 
- Regardless of the position taken by a clinician in regards to the above factors, the current UK law on consent requires that a full and detailed discussion about the indications for, efficacy of, and types of drugs used in spinal injections needs to occur and be documented.

- Most people will receive these injections for acute or chronic spinal pain probably associated with radicular pain such as brachialgia (arm pain) or sciatica (leg pain), and it is likely that these types of pain tend over time to recur and, if already chronic, will most likely recur whatever therapy is offered. It is more likely than not that the injection will help in the short term (days to months) in relieving symptoms and may shorten an attack of pain, so in effect 'curing that episode of pain', but most studies suggest that such injections are unlikely to change the long-term clinical course of pain and disability, or there is no high quality evidence to support such a change. ${ }^{27}, 28$, 29

In conclusion, the current evidence suggests steroids in general may be a useful addition to epidural blockade but particulate steroids are likely to be associated with an increased risk of catastrophic complications compared to non-particulate corticosteroid preparations, especially with neuroforaminal injections in the cervical region and more generally in the neuraxsis after spinal surgery. Non-particulate corticosteroids are probably going to work as well as particulate formulations, but there is no high-quality evidence that any corticosteroid-based axial spinal procedures in themselves are likely to change the long-term clinical outcome in an individual patient. Once the issue of fully informed patient involvement in the consenting process is also considered, the future role of any corticosteroid-based treatments in the treatment of axial spinal derived pain is likely to be diminished.

In our view some of the main issues that need to be addressed with the patient during the consenting process for pain-related procedures are given in appendix 1 , an issue also discussed elsewhere. ${ }^{30}, 31,32$ However, the exact issues discussed will be those relevant to the particular case and individual patient and therefore this list will need to be adapted on a case by case basis and cannot be a list of every issue which might need to be considered. We recommend that a patient information leaflet or similar be considered to address these issues prior to a final discussion about whether to proceed with a spinal injection. 


\section{Appendix 1}

List of possible questions that should be addressed during the consenting process for an axial spinal procedure

1) What is the natural history of this spinal condition? In particular, what are the chances that my condition will improve or deteriorate without this spinal injection and what are likely to be the implications of that for me?

2) What are the risks of a) minor complications (short-term non-life changing effects) b) major complications (life changing effects, including rare catastrophic neurological complications) as a result of the injection?

3) What difference does the addition of corticosteroid make to the efficacy of this injection?

4) Does the use of a non-particulate over particulate corticosteroid make the injection safer, and if so, by how much?

5) Does the use of a non-particulate over particulate corticosteroid make the spinal injections less effective?

6) How do the risks and potential benefits of the injection compare if I have no corticosteroid in it at all?

7) Are cervical injections more dangerous than injections in the thoracic and lumbar region? If so why, and what difference does the use of non-particulate corticosteroids make to that risk?

8) Are there alternatives to this invasive injection such as other oral analgesics or oral corticosteroids, how effective are they likely to be, and what possible complications might there be in such treatment?

9) Will this injection work for me a) in the short term (i.e. days to weeks and perhaps months) and b) in the long term (i.e. many months to years or permanently)? Alternatively, following a spinal injection will my spinal condition recur, and if so, over what period of time?

10) If this injection works for me, will I need it again?

11) If this injection does work initially but the benefit is lost over time, is this injection simply delaying a definitive treatment (such as surgery) which I would require in any case?

12) What are the risks of surgery compared to these injections, and how likely is surgery to be an effective (long-term) mode of treatment? 
13) Are there safer ways of performing the injections, and does the use of contrast and imaging make a difference to the risk?

14) Does the presence of other agents such as preservative in the corticosteroid preparation have any possible adverse effect on me?

15) Does the use of preservative-free injections mean that I am more likely to get an infection from a possible contaminated batch of drug?

\section{References}

1. Rathmell JP, Benzon HT, Dreyfuss P, et al. Safeguards to Prevent Neurologic Complications after Epidural Steroid Injections: Consensus Opinions from a Multidisciplinary Working Group and National Organizations. Anesthesiology 2015; 122: 974-984.

2. Benzon HT, Chew TL, McCarthy RJ, et al. Comparison of the particle sizes of different steroids and the effect of dilution: A review of the relative neurotoxicities of the steroids. Anesthesiology 2007; 106: 331-8.

3. Derby R, Lee SH, Date ES, et al. Size and aggregation of corticosteroids used for epidural injections. Pain Medicine 2008; 9: 227-38.

4. Dawley JD, Moeller-Bertram T, Wallace MS, et al. Intra-arterial injection in the rat brain: Evaluation of used for transforaminal epidurals. Spine 2009; 34: 1638-48.

5. Okubadejo G, Talcott MR, Schmidt RE, et al. Perils of intravascular methylprednisolone injection into the vertebral artery. Journal of Bone and Joint Surgery American 2008; 90:1932-8.

6. Lee JH, Lee JK, Seo BR, et al. Spinal cord injury produced by direct damage during cervical transforaminal epidural injection. Regional Anesthesia \& Pain Medicine 2008; 33: 377-9.

7. Rozin L, Rozin R, Koehler SA, et al. Death during transforaminal epidural steroid nerve root block (C7) due to perforation of the left vertebral artery. American Journal of Forensic Medicine and Pathology 2003; 2: $351-5$. 8. Brouwers PJ, Kottink EJ, Simon MA, et al. A cervical anterior spinal artery syndrome after diagnostic blockade of the right C6-nerve root. Pain 2001; 91: 397-399. 
9. MacVicar J, King W, Landers MH, et al. The Effectiveness of Lumbar Transforaminal Injection of Steroids: A comprehensive review with systematic analysis of the published data. Pain Medicine 2013; 14: 14-28. 10. Park CH, Lee SH and Kim BI. Comparison of the effectiveness of lumbar transforaminal epidural injection with particulate and nonparticulate corticosteroids in lumbar radiating pain. Pain Medicine 2010; 11: 16541658.

11. Kim D and Brown J. Efficacy and safety of lumbar epidural dexamethasone versus methylprednisolone in the treatment of lumbar radiculopathy: a comparison of soluble versus particulate steroids. Clinical Journal of Pain 2011; 27: 518-22.

12. Feeley IH, Healy EF, Noel J, et al. Eur Spine J. 2017 Feb;26(2):336-344. doi: 10.1007/s00586-016-4437-0. Epub 2016 Feb 12.

Particulate and non-particulate steroids in spinal epidurals: a systematic review and meta-analysis. $\mathrm{M}$

13. El-Yahchouchi C, Geske JR, Carter RE, et al. The noninferiority of the nonparticulate steroid dexamethasone and triamcinolone in lumbar transforaminal epidural steroid injections. Pain Medicine 2013; 14: 1650-7. 14. Kennedy DJ, Plastaras C, Casey E, et al. Comparative effectiveness of lumbar transforaminal epidural steroid injections with particulate versus nonparticulate corticosteroid for lumbar radicular pain due to intervertebral disc herniation: a prospective, randomized, double-blind trial. Pain Medicine 2014:15: 548-555. 15. Fitzgibbon DR, Posner KL, Domino KB, et al. American Society of Anesthesiologists. Chronic pain management: American Society of Anesthesiologists Closed Claims Project. Anesthesiology 2004; 100: 98-105. 16. Rathmell JP, Michna E, Fitzgibbon DR, et al. Injury and liability associated with cervical procedures for chronic pain. Anesthesiology 2011; 114: 918-26.

17. Smith RM, Schaefer MK, Kainer MA, et al. Multistate Fungal Infection Outbreak Response Team. Fungal infections associated with contaminated methylprednisolone injections. N Engl J Med. 2013; 369: 1598-609. 18. Manchikanti L, Nampiaparampil DE, Manchikanti KN, et al. Comparison of the efficacy of saline, local anesthetics, and steroids in epidural and facet joint injections for the management of spinal pain: A systematic review of randomized controlled trials. Surg Neurol Int. 2015; 6 (Suppl 4): S194-235.

19. British Pain Society/Faculty of Pain Medicine (RCA) Consensus Statement on the use of Corticosteroids for Neuraxial Procedures in the UK https://www.britishpainsociety.org/static/uploads/resources/files/BPS_FPM_Steroid_Statement_FINAL.pdf 20. GMC June 2008 Consent Patients and Doctors making decisions together https://www.gmc-uk.org/ethicalguidance/ethical-guidance-for-doctors/consent

21. https://www.gmc-uk.org/about/get-involved/consultations/review-of-our-consent-guidance

22. Bolam v Friern Hospital Management Committee [1957] 2 All ER 118

23. Bolitho v City and Hackney Health Authority [1997] 3 WLR 1151

24. Montgomery v Lanarkshire Health Board [2015] UKSC 11

25. Sokol DK. Update on the UK law on consent. BMJ 2015;350:h1481.

26. Jones v Royal Devon and Exeter NHS Foundation Trust (Lawtel 22 September 2015).

27. Low back pain and sciatica in over $16 \mathrm{~s}$ : assessment and management

NICE guideline [NG59]Published date: November 2016 https://www.nice.org.uk/guidance/ng59

28. Neck pain - cervical radiculopathy NICE CKS https://cks.nice.org.uk/neck-pain-cervicalradiculopathy\#!topicSummary

29. Andrew Engel, MD, Wade King, MMedSc MMed(Pain), John MacVicar, MB ChB MPainMed Standards Division of the International Spine Intervention Society. The Effectiveness and Risks of Fluoroscopically Guided Cervical Transforaminal Injections of Steroids: A Systematic Review with Comprehensive Analysis of the Published Data. Pain Medicine, Volume 15, Issue 3, March 2014, Pages 386-402.

30. Munglani R, Eyre G, and Sharma M. Consent in Pain Medicine: Law and implications for practise. Pain News 2018; Vol 16(2): 60-65.

31. Munglani R, Eyre G and Sharma M. Consent in Pain Medicine: Law and implications for practise. Part 2 What does Valid Consent look like? Pain News 2018; Vol 16(3): 121-126.

32. Sharma M, Knagg R, Munglani R et al. Consent in Pain Medicine: Law and implications for practise. Part 3: the practicalities of consent in patients with chronic pain? Pain News 2018; Vol 16(4): 185-194. 


\section{British Journal of Pain Declarations Form}

A declarations form must be submitted for ALL types of manuscript. BJP operates a double-blind peer review process, so please do not include names of any authors or institutions in this form as it will be seen by peer-reviewers.

The $\boldsymbol{B J P}$ declaration policy document should be read before filling in this form. This provides options for many of the declarations below.

Title of Manuscript: Particulate steroids in axial spinal blockade and the increasing role of patient consent: Les grains de sable dans l'engrenage

Declaration of Conflicting Interest: The authors declare that there is no conflict of interest

Funding: This research received no specific grant from any funding agency in the public, commercial, or notfor-profit sectors.

Ethical approval: Not applicable. Review and consensus opinion.

Informed Consent: Not applicable. Review and consensus opinion.

Guarantor: RM

Contributorship: RM and RDK researched literature and conceived the review. RM wrote the first draft of the manuscript. All authors reviewed and edited the manuscript and approved the final version of the manuscript Acknowledgements: None

NOTE: Please do not leave any blank spaces. Appropriate wording can be found in the $\boldsymbol{B J P}$ declaration policy document

* The guarantor is the person willing to take full responsibility for the article, including for the accuracy and appropriateness of the reference list. This will often be the most senior member of the research group and is commonly also the author for correspondence. Please use initials only: BJP operates a double-blind peer review process so full names of authors should not be listed on this form. 